\title{
Functionalization of Hollow Mesoporous Silica Nanoparticles for Improved 5-FU Loading
}

\author{
Xiaodong She, Lijue Chen, Chengpeng Li, Canzhong He, Li He, and Lingxue Kong
}

Institute for Frontier Materials, Deakin University, Waurn Ponds, VIC 3216, Australia

Correspondence should be addressed to Lingxue Kong; lingxue.kong@deakin.edu.au

Received 4 December 2014; Revised 26 February 2015; Accepted 5 March 2015

Academic Editor: Andrea Falqui

Copyright (C) 2015 Xiaodong She et al. This is an open access article distributed under the Creative Commons Attribution License, which permits unrestricted use, distribution, and reproduction in any medium, provided the original work is properly cited.

\begin{abstract}
Hollow mesoporous silica nanoparticles were successfully fabricated and functionalized with appropriate silanes. After modifications, amine, carboxyl, cyano, and methyl groups were grafted onto the nanoparticles and all functionalized hollow mesoporous silica nanoparticles maintained a spherical and hollow structure with a mean diameter of $\sim 120 \mathrm{~nm}$ and a shell thickness of $\sim 10 \mathrm{~nm}$. The loading capacity of the hollow mesoporous silica nanoaprticles to the anticancer drug, 5-fluorouracil, can be controlled via precise functionalization. The presence of amine groups on the surface of nanoparticles resulted in the highest loading capacity of $28.89 \%$, due to the amine functionalized nanoparticles having a similar hydrophilicity but reverse charge to the drug. In addition, the change in $\mathrm{pH}$ leads to the variation of the intensity of electrostatic force between nanoparticles and the drug, which finally affects the loading capacity of amine functionalized hollow mesoporous silica nanoparticles to some extent. Higher drug loading was observed at $\mathrm{pH}$ of 7.4 and 8.5 as 5 -fluorouracil becomes more deprotonated in alkaline conditions. The improved drug loading capacity by amine functionalized hollow mesoporous silica nanoparticles has demonstrated that they can become potential intracellular 5fluorouracil delivery vehicles for cancers.
\end{abstract}

\section{Introduction}

Nanoparticle based targeted drug delivery systems hold great promise for cancer therapy because of their targeting functions [1], sustained drug release profiles [2], reduced side effects [3], and the ability to overcome multidrug resistance (MDR) $[2,4]$. A variety of materials have been exploited to meet different therapeutic needs, many with great potential for topical delivery [5-7]. When compared to polymer and carbon nanotubes which have been widely investigated as carriers in drug delivery systems [8-11], mesoporous silica nanoparticles (MSNs) have the potential to serve as a versatile drug nanocarrier for smart drug delivery [12]. For example, MSNs can level off the drug concentration at the targeted area as the drug molecules are released from the ordered pores by a tunable diffusion. This will reduce drug dosage and side effects [13]. Furthermore, MSNs are biocompatible, providing additional advantages for MSNs to be used as a platform for cancer treatment [14]. Choi et al. functionalized MSNs for the purpose of controlling drug release and developed a drug delivery system using MSNs as carriers based on the hostguest concept [15]. Mamaeva and coworkers [16] introduced a MSNs platform for cancer treatments. In their study, MSNs based drug delivery system can block the Notch signaling in cancer by controlling the delivery of $\gamma$-secretase inhibitors to cancer cells. Zhao et al. constructed a drug delivery system with sustained-release property based on rattle-type hollow magnetic mesoporous spheres and achieved an ibuprofen loading capacity of $302 \mathrm{mg} \mathrm{g}^{-1}$ [17].

However, even though with a high surface area and a high pore volume, MSNs showed a relative low loading capacity of 5-fluorouracil (5-FU), a standalone therapeutic for the treatment of colon cancer, which may be due to the electrostatic repulsion between the drug and the carriers. In this case, in comparison to those carriers with a higher drug loading capacity, more MSNs were required to be taken up by cancer cells to achieve an effective cure concentration for cancer therapy. From a technical point of view, it is more preferable to deliver a smaller quantity of MSNs to minimise the side effects to patients [18]. Therefore, increasing the drug 
loading capacity of MSNs is essential for further optimizing the MSN based 5-FU delivery system.

The capacity to encapsulate drugs in nanoparticles can be improved by functionalizing or optimizing the drug encapsulation conditions [19]. Precisely controlling the grafting of chemical groups on MSNs would result in an enhancement in drug loading. For example, Sevimli and Yilmaz grafted primary amine, organothiol, and methyl groups onto MSNs via different alkoxysilanes and found that the amount of amoxicillin loaded was depended on the type of grafted functional groups [20]. Tomoiaga and coworkers functionalized magnetic MSNs with amino groups and achieved improved 5-FU loading capacity when compared to plain MSNs [21]. On the other hand, hollow MSNs (HMSNs) were reported to encapsulate more drugs than nonhollow ones as the hollow cavity provided more space for the storage of the drugs [22-24].

In this paper, HMSNs were modified for enhanced 5-FU loading. The partition coefficient of 5-FU was investigated to provide information about the degree of the hydrophilicity of 5-FU, which is crucial for selecting chemical groups for the subsequent functionalization. Amine, carboxyl, methyl, and cyano groups were introduced onto HMSNs. The structure of plain and functionalized HMSNs was characterized and the 5-FU loading capacity of HMSNs with and without chemical groups was investigated and compared.

\section{Materials and Methods}

2.1. Materials. Tetraethyl orthosilicate (TEOS, $\left.\mathrm{Si}\left(\mathrm{OCH}_{2} \mathrm{CH}_{3}\right)_{4}\right)$, Triton X-100 (TX100), sulphuric acid, octadecyltrimethoxysilane (OTMS), (3-aminopropyl)triethoxysilane (APTES), 3cyanopropyltriethoxysilane (CPTES), and fluorinated pyrimidine 5-fluorouracil (5-FU) were all purchased from Sigma Aldrich (Sydney, Australia). Eudragit S100 which is made in Germany was purchased from Shenzhen Youpuhui Pharmaceutical Co. Ltd (Shenzhen, China).

2.2. Methods and Characterizations. For the formation of the mesoporous silica shell, the Eudragit S-100 nanoparticle solution was ultrasonically dispersed for $3 \mathrm{~min}$, followed by addition of $1.0 \mathrm{~g}$ TX100 under stirring. After TX100 was completely dissolved, $2.3 \mathrm{~mL}$ TEOS was added and this solution was left to proceed for $24 \mathrm{~h}$ before being transferred into a sealed container and hydrothermally treated overnight under $100^{\circ} \mathrm{C}$. The resulting nanoparticles (as-synthesized HMSNs) were collected by centrifugation and redispersed in acetone for the removal of Eudragit core and TX100 templates. The particles were then washed 3 times with ethanol and deionized water, respectively, before finally freeze-drying.

For amine functionalization $\left(\mathrm{HMSN}-\mathrm{NH}_{2}\right), 50 \mathrm{mg}$ HMSNs were ultrasonically dispersed in $50 \mathrm{~mL}$ ethanol; $25 \mu \mathrm{L}$ APTES was added and stirred for $24 \mathrm{~h}$ before centrifugations and washed 3 times with ethanol and then deionized water, respectively. Finally these yielded $\mathrm{HMSN}-\mathrm{NH}_{2}$ nanoparticles were collected and freeze-dried. The experimental procedure for methyl- and cyanofunctionalization ( $\mathrm{HMSN}-\mathrm{CH}_{3}$ and HMSN-CN) is similar to amine functionalization. The only difference is to use OTMS and CPTES instead of APTES for chemical modification.
To prepare carboxyl-modified MSNs (HMSN-COOH), fifty milligrams of HMSNs was ultrasonically dispersed in $50 \mathrm{~mL}$ ethanol; $25 \mu \mathrm{L}$ CPTES was added and stirred for $24 \mathrm{~h}$. The resulting nanoparticles were collected by centrifugations and redispersed in sulphuric acid $(50 \% \mathrm{v} / \mathrm{v})$ and heated at $150^{\circ} \mathrm{C}$ for $4 \mathrm{~h}$. The final products were recollected, washed, and freeze-dried.

Measurement of 5-FU oil: water partition coefficients was conducted via shake flash method according to OECD guideline 107 [25]. Firstly, n-octanol and Milli-Q water were saturated, respectively, prior to the experiments and herein their saturated solutions were referred to as octanol and water. Then, 5-FU was dissolved in water to obtain a concentration of $266.7 \mu \mathrm{g} / \mathrm{mL}$. A combination of octanol and water with an octanol: water ratio of $1: 1,1: 2$, and $2: 1$ was added to centrifuge tubes and a total volume of $40 \mathrm{~mL}$ was maintained. Each ratio was carried out in triplicate. $1 \mathrm{~mL} 5-\mathrm{FU}$ was added to the centrifuge tubes and the phases were mixed by vortex for $10 \mathrm{~min}$ before centrifuging at $1000 \mathrm{rpm}$ for a further $10 \mathrm{~min}$. Both 5-FU concentrations in water and octanol phases were determined via UV-Vis spectroscopy (Ocean Optics system, USA). The partition coefficient $P_{\text {ow }}$ is calculated from the data of each run using the following equation:

$$
P_{\text {ow }}=\frac{C_{\text {n-octonal }}}{C_{\text {water }}}
$$

The loading of 5-FU was carried out by soaking HMSNs in a concentrated drug solution at room temperature for $24 \mathrm{~h}$ while stirring to ensure the diffusion of the drug molecules through the mesopores. Firstly, 5-FU was dissolved in deionized water to a concentration of $3 \mathrm{mg} / \mathrm{mL} .150 \mathrm{mg}$ of HMSNs was ultrasonically dispersed in $50 \mathrm{~mL}$ of the 5FU solution. The mixture was stirred at room temperature. At timed intervals of $0,2,4,6,8,12$, and $24 \mathrm{~h}, 0.5 \mathrm{~mL}$ of the solution was extracted and centrifuged to collect the supernatant for UV-Vis analysis at a wavelength of $266 \mathrm{~nm}$ while the 5-FU-loaded HMSNs precipitate was placed back into the drug solution. The 5-FU loading in the HMSNs was calculated by subtracting the amount of 5-FU in the supernatant from the amount in the original drug loading solution. The drug loading capacity was calculated using the following equation:

$$
\text { Loading capacity }(w t \%)=\frac{\text { Mass of drugs in the HMSNs }}{\text { Initial Mass of HMSNs }} \text {. }
$$

The morphology of HMSNs was studied using scanning electron microscopy (SEM, Carl Zeiss AG SEM Supra 55VP) and transmission electron microscopy (TEM, LaB6 JEOL JEM-2100). Before imaging under SEM, all the samples were coated with carbon through a BAL-TEC SCD 050 sputter coater (Leica Microsystems, Australia). TEM imaging was conducted under $200 \mathrm{kV}$. Nitrogen absorption and desorption isotherms of HMSNs were conducted on a Micromeritics Tristar 3000 (Particle \& Surface Science, UK) equipment at $77 \mathrm{~K}$. Before measurements, samples were degassed at $523 \mathrm{~K}$ for $1 \mathrm{~h}$ under nitrogen. The specific surface areas of 
HMSNs were calculated using the Brunauer-Emmett-Teller (BET) method. The Barrett-Joyner-Halenda (BJH) model was utilized to obtain the pore size distributions from the desorption branch of isotherms. Thermogravimetric analysis (TGA) of HMSNs was conducted using a TG Simultaneous Thermal Analyser (STA 449C, ZETZSCH, Germany). Samples with a weight of $\sim 5 \mathrm{mg}$ were placed into aluminium oxide crucibles and heated under nitrogen condition at a heating rate of $10^{\circ} \mathrm{C} / \mathrm{min}$ from room temperature to $600^{\circ} \mathrm{C}$. Fourier transform infrared (FTIR) spectra were measured with a Vertex 70 spectrometer (Bruker, Germany). All the samples were compressed into $\mathrm{KBr}$ pellets and recorded at 64 scans from $4000 \mathrm{~cm}^{-1}$ to $400 \mathrm{~cm}^{-1}$ with a resolution of $4 \mathrm{~cm}^{-1}$.

\section{Results and Discussions}

3.1. Partition Coefficient of 5-FU. The partition coefficient $\left(P_{\mathrm{ow}}\right)$ is defined as the quotient of two concentrations of a test substance (like 5-FU) in a two-phase system, where two largely immiscible solvents (n-octanol and water) were mixed with a fixed volume ratio. The logarithm of $P_{\text {ow }}$ between noctanol and water $\left(\log P_{\text {ow }}\right)$ has often been used as a key parameter to describe the lipophilicity of a molecule. In the pharmaceutical field, $\log P_{\text {ow }}$ is utilized to estimate the biological activities of the drugs, such as solubility [26], biodegradation rate, bioaccumulation, membrane permeability [27], and drug absorption and toxicity predictions. Therefore, the investigation of the $P_{\text {ow }}$ of 5-FU is essential to predict its transport activity, thereby providing information for the subsequent modification of HMSNs specific for high 5-FU loading.

The shake flask method is one of the common and standard experimental procedures adopted for $\log P_{\text {ow }}$ study. This method is applied to determine the solubility and hydrophobicity of compounds with $\log P_{\text {ow }}$ values ranging from -2 to 4 . The determination is based on the principle that hydrophobic substances soluble in the lipid phase show a positive $\log P_{\text {ow }}\left(\log P_{\text {ow }}>0\right)$, while negative values $\left(\log P_{\text {ow }}<\right.$ $0)$ typify polar compounds soluble in the water phase.

According to OECD guideline 107 [25], an effective partition coefficient study refers to all $\log P_{\text {ow }}$ values obtained falling within a range of $-2 \sim 4$ with an offset of \pm 0.3 units. As shown in Table 1, the $\log P_{\text {ow }}$ values of 5-FU for all ratios (octanol: water) are within the range and are consistently negative, indicating that 5 -FU is hydrophilic. Specifically, when the volume of water decreased from $26.67 \mathrm{~mL}$ to $13.33 \mathrm{~mL}$, the 5 -FU concentration in water phase increased significantly from $7.747 \mu \mathrm{g} / \mathrm{mL}$ to $13.17 \mu \mathrm{g} / \mathrm{mL}$. This is due to the fact that most 5-FU molecules prefer congregating in the water phase; thus, with a decrease in the water volume, a more concentrated 5-FU solution is observed. In contrast, no obvious difference was found in 5-FU concentration in the octanol phase. With varying octanol volume, from $26.67 \mathrm{~mL}$ to $13.33 \mathrm{~mL}, 5-\mathrm{FU}$ concentration remained at around $2.2 \sim$ $2.6 \mu \mathrm{g} / \mathrm{mL}$, which can be explained by the hydrophilic property of 5 -FU.

The weight percentages of 5-FU dissolved in each phase with varying octanol to water ratio were calculated. As seen
TABLE 1: Partition coefficient of 5-FU and relevant parameters.

\begin{tabular}{lccc}
\hline & $1: 2(\mathrm{O}: \mathrm{W})$ & $1: 1(\mathrm{O}: \mathrm{W})$ & $2: 1(\mathrm{O}: \mathrm{W})$ \\
\hline Water volume $(\mathrm{mL})$ & 26.67 & 20 & 13.33 \\
Oil volume $(\mathrm{mL})$ & 13.33 & 20 & 26.67 \\
$C_{\text {water }}(\mu \mathrm{g} / \mathrm{mL})$ & 7.747 & 9.925 & 13.17 \\
$C_{\text {octanol }}(\mu \mathrm{g} / \mathrm{mL})$ & 2.279 & 2.380 & 2.629 \\
$P_{\text {ow }}$ & 0.2942 & 0.2398 & 0.1996 \\
$\log P_{\text {ow }}$ & -0.5314 & -0.6201 & -0.6997 \\
\hline
\end{tabular}

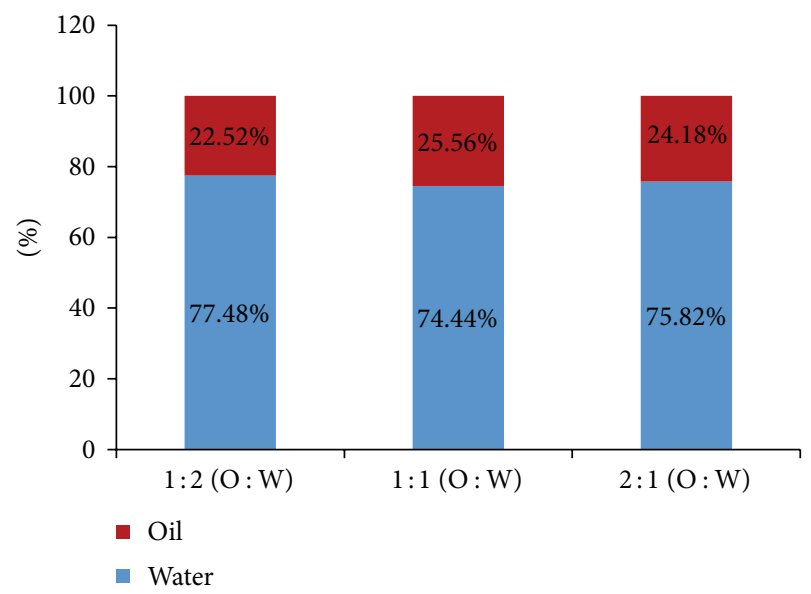

FIGURE 1: Percentages of 5-FU dissolved in each phase with varying octanol to water ratio.

in Figure 1, even though the volume of water and octanol changes, it appears that, in all ratios, around $75 \%$ of $5-\mathrm{FU}$ is dissolved in the water phase while the other $\sim 25 \%$ was found in the octanol phase. Therefore, a more hydrophilic surface of HMSNs would increase the loading of 5-FU.

3.2. Modification of HMSNs. Surface-functionalized HMSNs were synthesized by postgrafting method where chemical groups were introduced to the surface of as-synthesized HMSNs using appropriate silanes. CPTES, APTES, and TEMS were used to prepare HMSN-CN, HMSN-NH , and $\mathrm{HMSN}-\mathrm{CH}_{3}$ while $\mathrm{HMSN}-\mathrm{COOH}$ was obtained by oxidizing HMSN-CN. In all cases, plain HMSNs without any modification were used as a control. The morphology of modified and plain HMSNs was characterized by TEM. As shown in Figure 2, all modified HMSNs (Figures 2(b)-2(e)) had a similar morphology to that of plain HMSNs (Figure 2(a)) revealing that the structure of these nanoparticles can be well preserved during all functionalization processes. In particular, the surface-functionalized HMSNs maintained a hollow structure and a very spherical shape with a mean diameter of $\sim 120 \mathrm{~nm}$ and a shell thickness of $\sim 10 \mathrm{~nm}$. In addition, all HMSNs displayed a highly uniform size, shell thickness, and, more importantly, a good dispersion which is one of the main requirements for drug delivery systems.

The presence of mesoporous structure in all HMSNs was confirmed by BET (Figure 3 ). The nitrogen adsorption/desorption experiments demonstrated that both modified and plain HMSNs showed characteristic isotherms of 
TABLE 2: Structure parameters of functionalized and nonfunctionalized HMSNs.

\begin{tabular}{lccccc}
\hline HMSNs & HMSN-CH $_{3}$ & HMSN-CN & HMSN-NH & HMSN-COOH & Plain HMSNs \\
\hline Pore size $(\mathrm{nm})$ & 2.51 & 2.28 & 2.47 & 2.61 & 2.78 \\
Pore volume $\left(\mathrm{cm}^{3} / \mathrm{g}\right)$ & 0.43 & 0.50 & 0.43 & 0.47 & 0.53 \\
Surface area $\left(\mathrm{m}^{2} / \mathrm{g}\right)$ & 434 & 514 & 413 & 498 & 820 \\
\hline
\end{tabular}

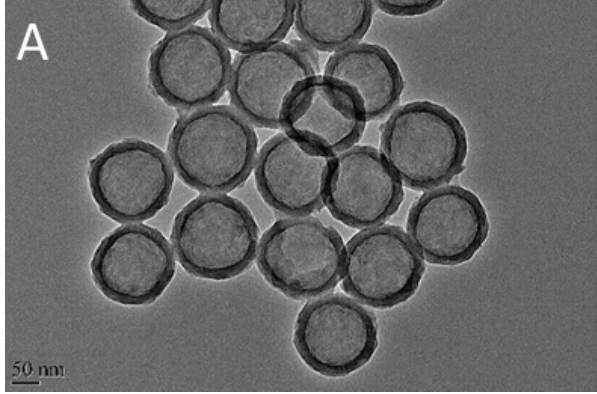

(a)

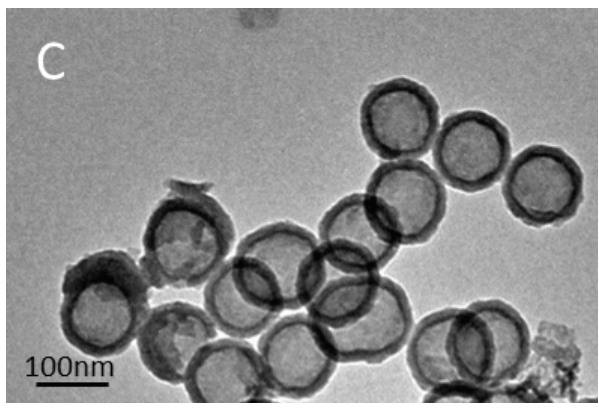

(c)

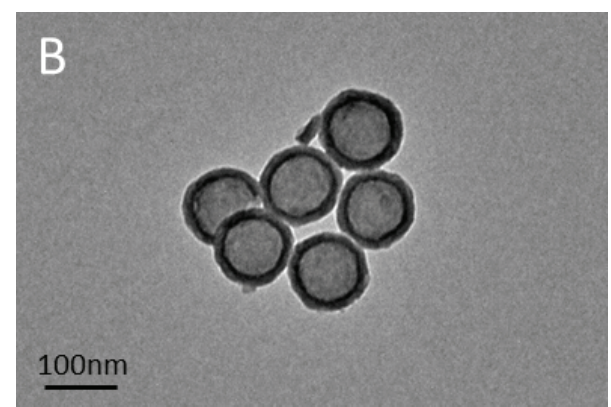

(b)

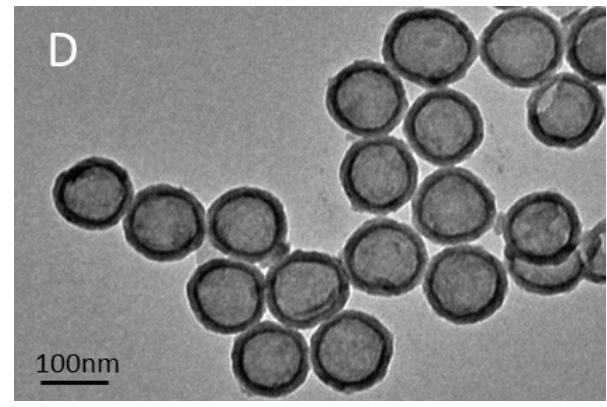

(d)

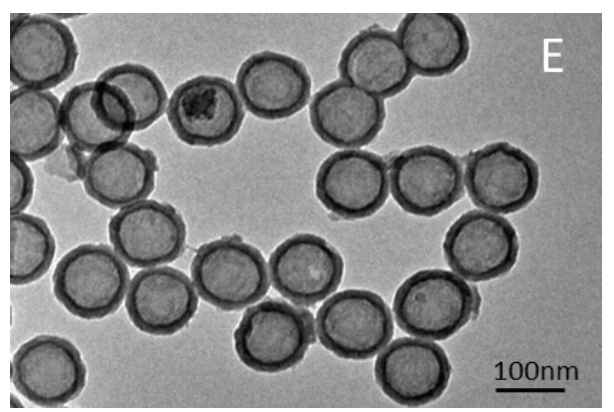

(e)

Figure 2: TEM images of (a) HMSNs; (b) HMSN-NH $\mathrm{H}_{2}$ ( (c) HMSN-COOH; (d) HMSN-CN; and (e) HMSN-CH

mesoporous materials, a type IV isotherm, indicating the existence of mesopores $[17,28,29]$. Well-defined steps which occurred in the relative pressure of $0.35-0.55$ and $0.9-1.0$ were related to capillary condensation and desorption in open mesopores and interstitial pores, respectively [30]. As seen in Figure 3(b), narrow pore size distributions are presented in HMSNs before and after surface modifications. The average pore size of all HMSNs was calculated using the desorption branch of the isotherm by the typical Barrett-Joyner-Halenda (BJH) method, according to which HMSNs, HMSN-NH $\mathrm{HMSN}-\mathrm{CN}, \mathrm{HMSN}-\mathrm{COOH}$, and $\mathrm{HMSN}-\mathrm{CH}_{3}$ have a pore size of $2.78 \mathrm{~nm}, 2.47 \mathrm{~nm}, 2.28 \mathrm{~nm}, 2.61 \mathrm{~nm}$, and $2.51 \mathrm{~nm}$, respectively.
Structural parameters of the HMSNs with and without modifications were summarized in Table 2. No significant differences can be found in the pore size and the pore volume between nanoparticles functionalized with different groups, proving that surface functionalization had a limited effect on the morphology and microstructure of the final products. The maintaining of pore size and pore volume guarantees large spaces in functionalized HMSNs for drug loading that ideally should be same as nonfunctionalized ones. However, due to the coverage of functional groups, the surface area of functionalized samples decreased when compared to plain HMSNs. These results were consistent with similar studies [20]. 


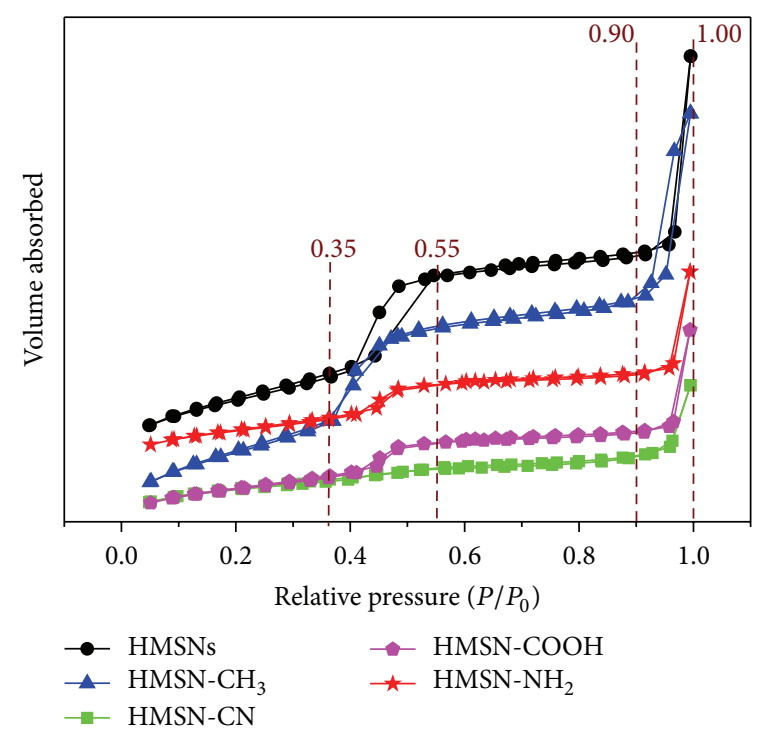

(a)

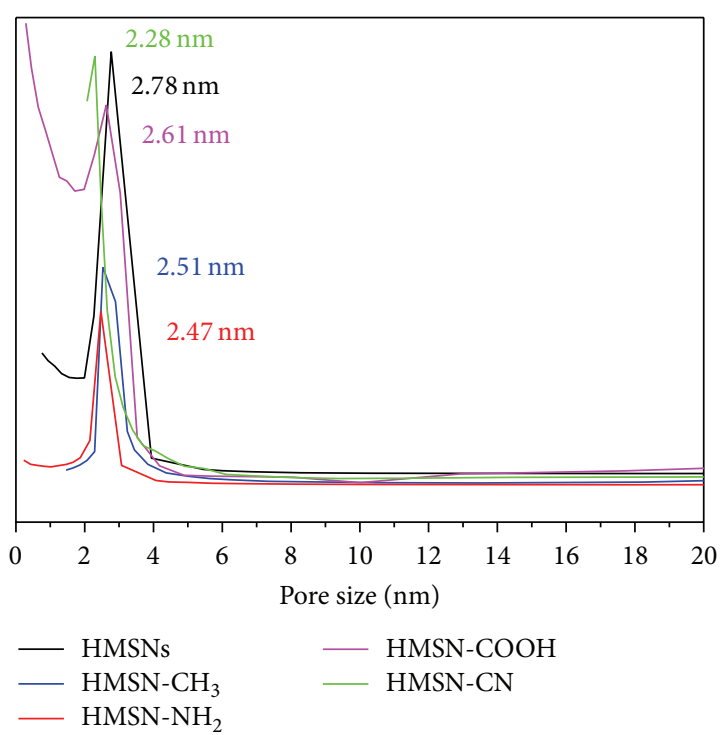

(b)

FIGURE 3: $\mathrm{N}_{2}$ adsorption-desorption isotherms (a) and the corresponding pore size distributions (b) of functionalized and plain HMSNs.

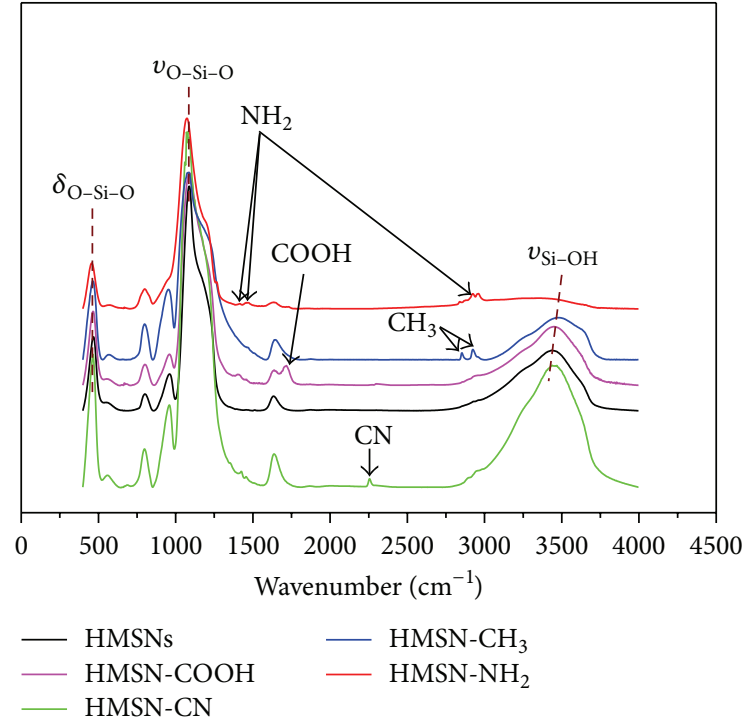

FIGURE 4: FTIR spectra of plain and functionalized HMSNs.

Different chemical groups of $-\mathrm{NH}_{2},-\mathrm{CN},-\mathrm{COOH}$, and $-\mathrm{CH}_{3}$ were grafted onto the surface of HMSNs separately and the successful grafting was demonstrated from FTIR spectra (Figure 4). All samples have strong bands located at around $460\left(\delta_{\mathrm{O}-\mathrm{Si}-\mathrm{O}}\right), 1100\left(v_{\text {asym.Si-O-Si }}\right)$, and $3300-$ $3500 \mathrm{~cm}^{-1}\left(v_{\mathrm{Si}-\mathrm{OH}}\right)$, confirming the presence of the $\mathrm{SiO}_{2}$ inorganic phase [31]. The $-\mathrm{NH}_{2}$ functionalization was clearly evidenced by the bands detected at around $1500 \mathrm{~cm}^{-1}$ and $3000 \mathrm{~cm}^{-1}$, which were unambiguously attributed to the $\mathrm{NH}$ bending vibration and $\mathrm{C}-\mathrm{N}$ stretching vibration, respectively [20, 31-34]. Similarly, $-\mathrm{CN}$ and $-\mathrm{COOH}$ functionalizations were proved by the observation of relative characteristic peaks. The peak at $2200 \mathrm{~cm}^{-1}$ corresponding to cyano groups $(\mathrm{CN})$ was observed in the spectrum of HMSN-CN while HMSN-COOH showed a peak at $1730 \mathrm{~cm}^{-1}$ relating to $\mathrm{C}=\mathrm{O}$ vibration of $\mathrm{COOH}$. The bands appearing at around $3000 \mathrm{~cm}^{-1}$ in the spectrum of $\mathrm{HMSN}-\mathrm{CH}_{3}$ were assigned to $\mathrm{C}-\mathrm{H}$ vibration of methyl groups $\left(-\mathrm{CH}_{3}\right)$, confirming the existence of $-\mathrm{CH}_{3}$ on the surface of $\mathrm{HMSN}-\mathrm{CH}_{3}$. Results obtained by FTIR proved the effective surface functionalization of HMSNs, thereby confirming the presence of $-\mathrm{NH}_{2}$, $-\mathrm{CN},-\mathrm{COOH}$, and $-\mathrm{CH}_{3}$ on the surface of HMSN-NH $\mathrm{N}_{2}$, $\mathrm{HMSN}-\mathrm{CN}, \mathrm{HMSN}-\mathrm{COOH}$, and $\mathrm{HMSN}-\mathrm{CH}_{3}$, respectively.

Thermogravimetric analysis (TGA) was carried out to further confirm the effective surface modification of functionalized HMSNs. All samples were tested under nitrogen condition at the temperature ranging from room temperature up to $650^{\circ} \mathrm{C}$. The weight loss of samples obtained from TGA arose from the decomposition of chemical groups; thereby a difference in the weight loss between samples can indicate the relative amount of chemical groups grafted onto particles. As seen from Figure 5, the total weight loss of plain HMSNs was $\sim 8.0 \%$ due to the dehydroxylation of silanol groups $(\mathrm{Si}-\mathrm{OH})$. In contrast, all functionalized HMSNs showed a total weight loss of $\sim 20 \%$ which was mainly from thermal cracking of grafted functional groups. Therefore, the success of the grafting has been proven by TGA results, which was in agreement with the FTIR results illustrated above and the relative amount of functional groups of modified HMSNs was $\sim 12 \%(\mathrm{w} / \mathrm{w})$. Compared to other studies conducting functionalization on MSNs [20,35], the weight loss caused by the functional groups reported here is slightly higher, suggesting the high efficiency of the current functionalization. Modifications. The 5-FU loading capacities of functionalized 
TABLE 3: Loading capacities of functionalized and nonfunctionalized HMSNs.

\begin{tabular}{lccccc}
\hline HMSNs & HMSN-CH $_{3}$ & HMSN-CN & HMSN-NH $_{2}$ & HMSN-COOH & Plain HMSNs \\
\hline Loading capacity (mg 5-FU/g HMSNs) & 127.3 & 225.4 & 288.9 & 207.3 & 183.4 \\
\hline
\end{tabular}

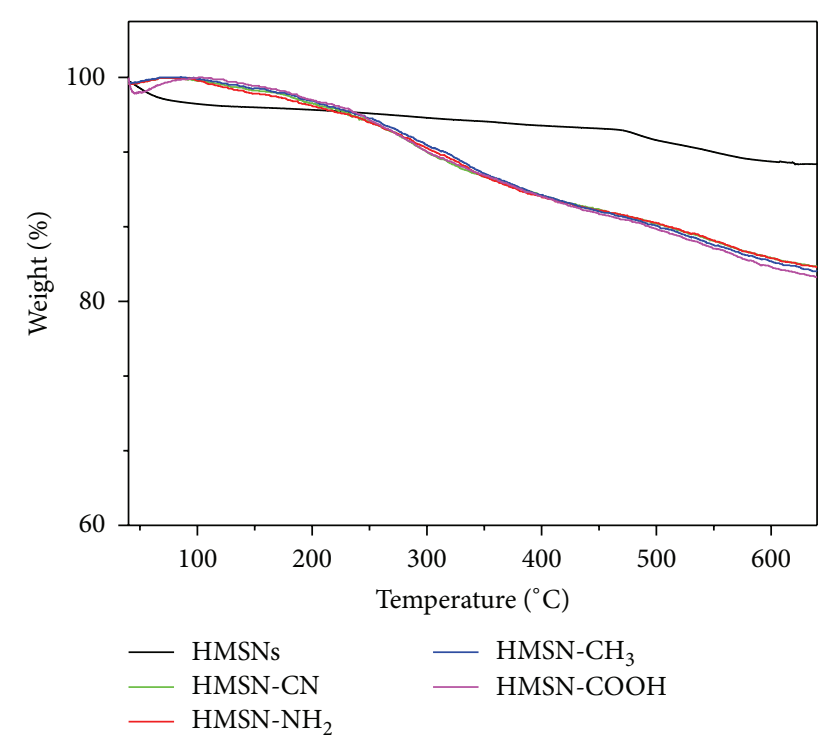

FIgURE 5: TGA curves of functionalized HMSNs and plain HMSNs.

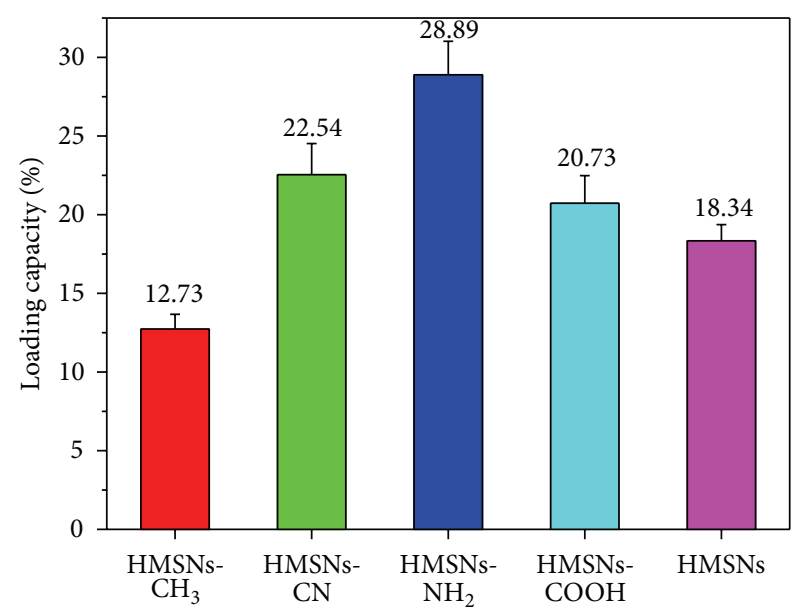

FIGURE 6: Loading capacities of functionalized and nonfunctionalized HMSNs.

and nonfunctionalized HMSNs have been monitored using a UV spectrophotometer. An initial drug concentration of $3 \mathrm{mg} / \mathrm{mL}$ was applied and the concentration change after 24 hours of loading was tracked. The loading capacity of all HMSNs were calculated and presented in Figure 6. For HMSNs, the internal macropore (the hollow cavity) is used to carry the loaded drug, while the mesopores in the shell are applied for drug storage as well as for transportation and controlled release of drugs from the macropore.

5 -FU was absorbed in both the pores and the hollow cavity and the external surface area. Firstly, 5-FU is attached

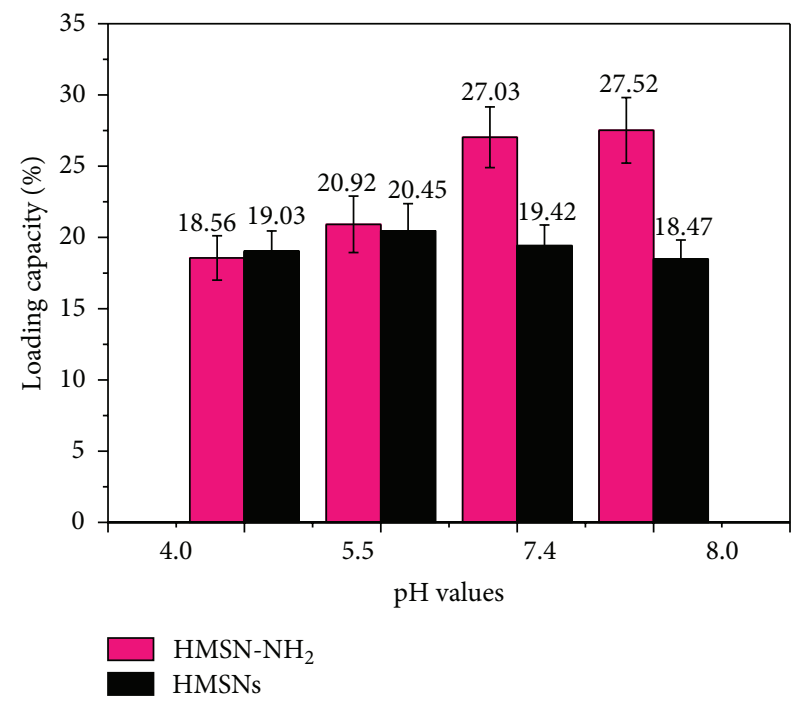

FIgURE 7: 5-FU loading capacity of HMSNs and $\mathrm{HMSN}_{-} \mathrm{NH}_{2}$ at different $\mathrm{pH}$ values.

on the surface of nanoparticles; as the loading process goes on, 5 -FU can pass through the pores $(2-3 \mathrm{~nm})$ and get into the cavity $(\sim 100 \mathrm{~nm})$. As the pores are much bigger than the drug molecules $(\sim 0.5 \mathrm{~nm})$ and can provide enough room for the free transportation of the drug, the drug can be encapsulated into the pores and the cavity of HMSNs.

The presence of different functional groups on the external area of nanoparticles will affect the loading capacity of the nanoparticles. As expected, a difference in the amount of 5-FU loading after surface functionalization was observed, resulting from the different hydrophobicity and hydrophilicity of the functional groups [20]. A loading percentage of $18.34 \%$ was achieved for the plain HMSNs but increased to $28.89 \%$ after amine functionalization. It was reported that, by using amine functionalized mesoporous silica nanoparticles (MSNs, nonhollow structure) specifically for 5-FU encapsulation, a 5-FU loading of $124 \mathrm{mg} 5$-FU/g MSNs was achieved [21]. It is known that the degree and spatial distribution of the functionalities can affect the drug loading process. However, the hollow morphology is another parameter that could contribute to the high efficiency in drug loading of MSNs with a hollow morphology [22-24]. Therefore, this enhanced loading capacity (Table 3 ) might be attributed to the hollow structure of HMSNs and the functionalization.

Furthermore, $-\mathrm{CH}_{3}$ functionalization decreases the loading capacity of HMSNs to some extent (from 18.34\% to $12.73 \%) .5-\mathrm{FU}$ is a water-soluble drug; therefore, it is harder for it to approach HMSNs functionalized with methyl groups which are slightly more hydrophobic. On the other hand, $-\mathrm{CN}$ and $-\mathrm{COOH}$ functionalized HMSNs showed 


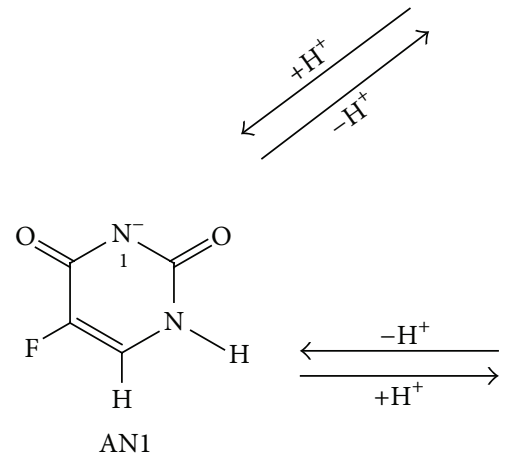<smiles>O=C1C=CC(=O)C(=O)[N-]1</smiles>

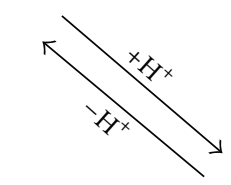

FIGURE 8: 5-FU anions (AN1 and AN3) and dianion.

a moderate improvement in loading with a loading percentage of $22.54 \%$ and $20.73 \%$, respectively. Although HMSN$\mathrm{COOH}$ is hydrophilic which is similar to $5-\mathrm{FU}$, its loading capacity is inferior to $\mathrm{HMSN}-\mathrm{NH}_{2}$. This can be explained by the different surface charge of $\mathrm{HMSN}-\mathrm{NH}_{2}$ and HMSN$\mathrm{COOH}$. Given that 5-FU is negatively charged, it is much easier for 5-FU to get inside the positively charged HMSN$\mathrm{NH}_{2}$ via electrostatic attractions. However, the negative charge of $-\mathrm{COOH}$ groups may repel 5-FU molecules and decrease the 5-FU loading capacity of HMSN-COOH.

3.4. The Effect of $p H$ on Drug Loading and Release from $\mathrm{HMSN}-\mathrm{NH}_{2}$. The $-\mathrm{NH}_{2}$ on HMSNs can significantly increase the amount of 5-FU encapsulated into nanoparticles due to the similar hydrophilicity of the HMSN-NH to drug and the existence of positive charge provided by $-\mathrm{NH}_{2}$ groups. Therefore, the control of the intensity of electrostatic force between nanoparticles and 5-FU may further regulate 5-FU loading capacity of particles.

In this regard, the loading of 5-FU by $\mathrm{HMSN}-\mathrm{NH}_{2}$ was carried out in 4 different pHs, 4.0, 5.5, 7.4, and 8.5 (Figure 7). As $\mathrm{pH}$ increases, the 5-FU loading capacity of $\mathrm{HMSN}-\mathrm{NH}_{2}$ increases and the maximum loading capacity of $27.52 \%$ is attained at $\mathrm{pH}$ of 8.5 . $\mathrm{HMSN}-\mathrm{NH}_{2}$ is positively charged at a wider $\mathrm{pH}$ range because of the protonation of the amine groups while, under different $\mathrm{pH}$ values, the behaviour of 5 -FU in terms of surface charge is different [36, 37]. As shown in Figure 8, the deprotonation of 5-FU can occur at either $\mathrm{N} 1$ or $\mathrm{N} 3$ or both to form the monoanions AN1 and $\mathrm{AN} 3$ and dianion under alkaline condition. It is reported that, at $\mathrm{pH} 7-10, \mathrm{AN} 1, \mathrm{AN} 3$, and dianion of 5-FU coexist as a mixture in aqueous medium $[36,37]$. Therefore, at acidic pHs (4.0 and 5.5), 5-FU becomes less deprotonated or nondeprotonated, which results in a very low surface charge of 5-FU. Therefore, lower drug loading capacity of HMSN-NH $\mathrm{N}_{2}(18.56 \%$ and $19.03 \%)$ was observed at these two pHs. Nevertheless, when $\mathrm{pH}$ increases to 7.4 and 8.5 , the deprotonation of 5 -FU enhances, thereby enhancing electrostatic attractions between $\mathrm{HMSN}-\mathrm{NH}_{2}$ and 5-FU.
Instead of being a neutral molecule in the medium, most 5FU molecules transfer to monoanions and dianion, which generates negative charges and leads to more 5-FU being encapsulated into the nanoparticles.

On the other hand, no significant differences can be seen in the loading capacity of HMSNs at various pHs (Figure 8). Due to the presence of negatively charged silanolate groups on the surface, the loading capacity of HMSNs was inferior to $\mathrm{HMSN}-\mathrm{NH}_{2}$ at all $\mathrm{pHs}$. The encapsulation of 5-FU by HMSNs might be mainly based on hydrogen bonding rather than electrostatic force. In addition, under alkaline pHs where 5FU possesses more negative charges, a small decrease in drug loading capacity of HMSNs was found. This could be due to the repulsive interaction between carriers and the drug.

\section{Conclusion}

HMSNs were successfully functionalized with appropriate silanes. After functionalization, HMSNs with different chemical groups on the surface maintained the intact hollow structure and no significant changes were found with the pore size and pore volume. However, due to the coverage of functional groups, the surface area of functionalized samples decreased when compared to plain HMSNs, which were consistent with similar studies. The surface modification of functionalized HMSNs was proven by FTIR and TGA to be effective and the relative amount of grafted functional groups was $\sim 12 \%(w / w)$. The loading capacity of HMSNs specific to 5 -FU can be improved via precise functionalization. The presence of $-\mathrm{NH}_{2}$ groups on the surface of nanoparticles resulted in the highest 5 -FU loading capacity of $28.89 \%$. The similar hydrophilicity of the HMSN-NH $\mathrm{H}_{2}$ and the drug and the presence of reverse charge of the drug give rise to this highest loading capacity of $\mathrm{HMSN}-\mathrm{NH}_{2}$. In addition, when compared to that of nonhollow MSNs reported in a very similar study, the loading amount of 5 -FU by $\mathrm{HMSN}-\mathrm{NH}_{2}$ was much higher, suggesting that, besides functionalization, 5-FU loading could be improved by designing and fabricating MSNs of different structure such as the hollow MSNs. 
The change in $\mathrm{pH}$ leads to the variation of the intensity of electrostatic force between nanoparticles and 5-FU, which finally affect the loading capacity of $\mathrm{HMSN}-\mathrm{NH}_{2}$ to some extent. Higher drug loading of $\mathrm{HMSN}-\mathrm{NH}_{2}$ was observed at $\mathrm{pH}$ of 7.4 and 8.5 due to the fact that 5 -FU becomes more deprotonated in alkaline conditions. By incorporating targeting molecules such as folic acid and epidermal growth factor (EGF), HMSN-NH${ }_{2}$ with improved loading of 5-FU will definitely render the current drug delivery system more versatile for applications in cancer therapy.

\section{Conflict of Interests}

The authors declare that there is no conflict of interests regarding the publication of this paper.

\section{References}

[1] J. M. Rosenholm, A. Meinander, E. Peuhu et al., "Targeting of porous hybrid silica nanoparticles to cancer cells," ACS Nano, vol. 3, no. 1, pp. 197-206, 2009.

[2] Y. Gao, Y. Chen, X. Ji et al., "Controlled intracellular release of doxorubicin in multidrug-resistant cancer cells by tuning the shell-pore sizes of mesoporous silica nanoparticles," ACS Nano, vol. 5, no. 12, pp. 9788-9798, 2011.

[3] Q. He, Z. Zhang, Y. Gao, J. Shi, and Y. Li, "Intracellular localization and cytotoxicity of spherical mesoporous silica nano-and microparticles," Small, vol. 5, no. 23, pp. 2722-2729, 2009.

[4] H. Meng, W. X. Mai, H. Zhang et al., "Codelivery of an optimal drug/siRNA combination using mesoporous silica nanoparticles to overcome drug resistance in breast cancer in vitro and in vivo," ACS Nano, vol. 7, no. 2, pp. 994-1005, 2013.

[5] K. Y. Cai, F. Jiang, Z. Luo, and X. Y. Chen, "Temperatureresponsive controlled drug delivery system based on titanium nanotubes," Advanced Engineering Materials, vol. 12, no. 9, pp. B565-B570, 2010.

[6] W. Fang, J. Yang, J. Gong, and N. Zheng, "Photo- and pHtriggered release of anticancer drugs from mesoporous silicacoated Pd@Ag nanoparticles," Advanced Functional Materials, vol. 22, no. 4, pp. 842-848, 2012.

[7] M. A. Casadei, G. Pitarresi, R. Calabrese, P. Paolicelli, and G. Giammona, "Biodegradable and $\mathrm{pH}$-sensitive hydrogels for potential colon-specific drug delivery: characterization and in vitro release studies," Biomacromolecules, vol. 9, no. 1, pp. 4349, 2008.

[8] B. Han, M. Zhang, T. Tang et al., "The long-term fate and toxicity of PEG-modified single-walled carbon nanotube isoliquiritigenin delivery vehicles in rats," Journal of Nanomaterials, vol. 2014, Article ID 257391, 8 pages, 2014.

[9] C. Manaspon, K. Viravaidya-Pasuwat, and N. Pimpha, "Preparation of folate-conjugated pluronic F127/chitosan core-shell nanoparticles encapsulating doxorubicin for breast cancer treatment," Journal of Nanomaterials, vol. 2012, Article ID 593878, 11 pages, 2012.

[10] C. Cheng, K. H. Müller, K. K. K. Koziol et al., "Toxicity and imaging of multi-walled carbon nanotubes in human macrophage cells," Biomaterials, vol. 30, no. 25, pp. 4152-4160, 2009.

[11] S. Fuchs, T. Kapp, H. Otto et al., "A surface-modified dendrimer set for potential application as drug delivery vehicles: synthesis, in vitro toxicity, and intracellular localization," Chemistry, vol. 10, no. 5, pp. 1167-1192, 2004.

[12] X. W. Lou, L. A. Archer, and Z. Yang, "Hollow micro-/nanostructures: synthesis and applications," Advanced Materials, vol. 20, no. 21, pp. 3987-4019, 2008.

[13] X. Mei, D. Chen, N. Li et al., "Hollow mesoporous silica nanoparticles conjugated with $\mathrm{pH}$-sensitive amphiphilic diblock polymer for controlled drug release," Microporous and Mesoporous Materials, vol. 152, pp. 16-24, 2012.

[14] Z. Ma, J. Bai, Y. Wang, and X. Jiang, "Impact of shape and pore size of mesoporous silica nanoparticles on serum protein adsorption and RBCS hemolysis," ACS Applied Materials and Interfaces, vol. 6, no. 4, pp. 2431-2438, 2014.

[15] Y. L. Choi, J. Jaworski, M. L. Seo, S. J. Lee, and J. H. Jung, "Controlled release using mesoporous silica nanoparticles functionalized with 18-crown-6 derivative," Journal of Materials Chemistry, vol. 21, no. 22, pp. 7882-7885, 2011.

[16] V. Mamaeva, J. M. Rosenholm, L. T. Bate-Eya et al., "Mesoporous silica nanoparticles as drug delivery systems for targeted inhibition of notch signaling in cancer," Molecular Therapy, vol. 19, no. 8, pp. 1538-1546, 2011.

[17] W. Zhao, H. Chen, Y. Li, A. Li, M. Lang, and J. Shi, "Uniform rattle-type hollow magnetic mesoporous spheres as drug delivery carriers and their sustained-release property," Advanced Functional Materials, vol. 18, no. 18, pp. 2780-2788, 2008.

[18] M. Sardan, A. Yildirim, D. Mumcuoglu, A. B. Tekinay, and M. O. Guler, "Noncovalent functionalization of mesoporous silica nanoparticles with amphiphilic peptides," Journal of Materials Chemistry B, vol. 2, no. 15, pp. 2168-2174, 2014.

[19] N. H. N. Kamarudin, A. A. Jalil, S. Triwahyono et al., "Role of 3aminopropyltriethoxysilane in the preparation of mesoporous silica nanoparticles for ibuprofen delivery: effect on physicochemical properties," Microporous and Mesoporous Materials, vol. 180, pp. 235-241, 2013.

[20] F. Sevimli and A. Yilmaz, "Surface functionalization of SBA-15 particles for amoxicillin delivery," Microporous and Mesoporous Materials, vol. 158, pp. 281-291, 2012.

[21] A. M. Tomoiaga, B. I. Cioroiu, V. Nica, and A. Vasile, "Investigations on nanoconfinement of low-molecular antineoplastic agents into biocompatible magnetic matrices for drug targeting," Colloids and Surfaces B: Biointerfaces, vol. 111, pp. 52-59, 2013.

[22] X. She, L. Chen, L. Velleman et al., "Fabrication of high specificity hollow mesoporous silica nanoparticles assisted by Eudragit for targeted drug delivery," Journal of Colloid and Interface Science, vol. 445, pp. 151-160, 2015.

[23] Y. Jiao, J. Guo, S. Shen et al., "Synthesis of discrete and dispersible hollow mesoporous silica nanoparticles with tailored shell thickness for controlled drug release," Journal of Materials Chemistry, vol. 22, no. 34, pp. 17636-17643, 2012.

[24] W. Zhao, M. Lang, Y. Li, L. Li, and J. Shi, "Fabrication of uniform hollow mesoporous silica spheres and ellipsoids of tunable size through a facile hard-templating route," Journal of Materials Chemistry, vol. 19, no. 18, pp. 2778-2783, 2009.

[25] Guideline for the Testing of Chemicals-Partition Coefficient ( $n$ Octanol/Water): Shake Flask Method, OECD (Organisation for Economic Cooperation and Development), Paris, France, 1995.

[26] A. Noble, "Partition coefficients (n-octanol-water) for pesticides," Journal of Chromatography, vol. 642, no. 1-2, pp. 3-14, 1993. 
[27] S. Sato, Y. Hirotani, N. Ogura, E. Sasaki, and S. Kitagawa, "Enhancing effect of N-dodecyl-2-pyrrolidone on the percutaneous absorption of 5-fluorouracil derivatives," Chemical and Pharmaceutical Bulletin, vol. 46, no. 5, pp. 831-836, 1998.

[28] J. Choi, Y. Han, S. Park, J. Park, and H. Kim, "Pore characteristics and hydrothermal stability of mesoporous silica: role of oleic acid," Journal of Nanomaterials, vol. 2014, Article ID 580347, 8 pages, 2014.

[29] C.-J. Tsou, Y. Hung, and C.-Y. Mou, "Hollow mesoporous silica nanoparticles with tunable shell thickness and pore size distribution for application as broad-ranging $\mathrm{pH}$ nanosensor," Microporous and Mesoporous Materials, vol. 190, pp. 181-188, 2014.

[30] X. Xia, P. Xu, H. Yu, and X. Li, "Resonant micro-cantilever chemical sensor with one-step synthesis of - $\mathrm{COOH}$ functionalized mesoporous-silica nanoparticles for detection of tracelevel organophosphorus pesticide," in Proceedings of the IEEE Sensors Conference, pp. 1-4, Taipei, Taiwan, October 2012.

[31] J. L. Blin, C. Gérardin, L. Rodehüser, C. Selve, and M. J. Stébé, "Influence of alkyl peptidoamines on the structure of functionalized mesoporous silica," Chemistry of Materials, vol. 16, no. 24, pp. 5071-5080, 2004.

[32] N. Shadjou and M. Hasanzadeh, "Amino functionalized mesoporous silica decorated with iron oxide nanoparticles as a magnetically recoverable nanoreactor for the synthesis of a new series of 2,4-diphenylpyrido[4,3-d]pyrimidines," RSC Advances, vol. 4, no. 35, pp. 18117-18126, 2014.

[33] M. Alesker, A. Heller, Z. Malik, I. Makarovsky, and J.-P. Lellouche, "Hybrid silica nanoparticles traceable by fluorescence and FT-IR spectroscopy: preparation, characterization and preliminary biological studies," Journal of Materials Chemistry, vol. 21, no. 29, pp. 10883-10893, 2011.

[34] X. She, C. He, Z. Peng, and L. Kong, "Molecular-level dispersion of graphene into epoxidized natural rubber: morphology, interfacial interaction and mechanical reinforcement," Polymer, vol. 55, no. 26, pp. 6803-6810, 2014.

[35] M. Moritz and M. Łaniecki, "SBA-15 mesoporous material modified with APTES as the carrier for 2-(3-benzoylphenyl)propionic acid," Applied Surface Science, vol. 258, no. 19, pp. 75237529, 2012.

[36] K. L. Wierzchowski, E. Litonska, and D. Shugar, "Infrared and ultraviolet studies on the tautomeric equilibria in aqueous medium between monoanionic species of uracil, thymine, 5fluorouracil, and other 2,4-diketopyrimidines," Journal of the American Chemical Society, vol. 87, no. 20, pp. 4621-4629, 1965.

[37] N. Markova, V. Enchev, and G. Ivanova, “Tautomeric equilibria of 5-fluorouracil anionic species in water," The Journal of Physical Chemistry A, vol. 114, no. 50, pp. 13154-13162, 2010. 

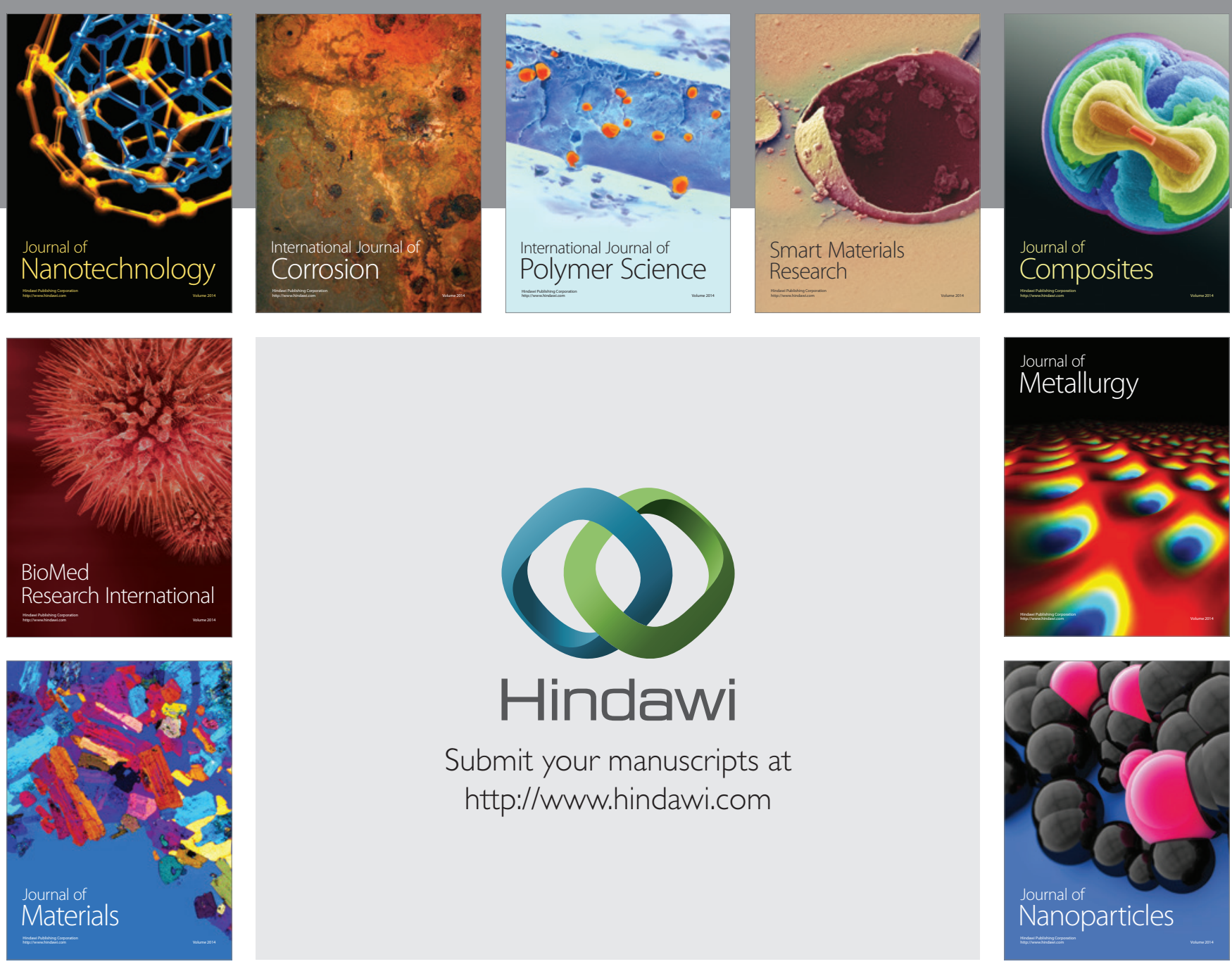

Submit your manuscripts at http://www.hindawi.com
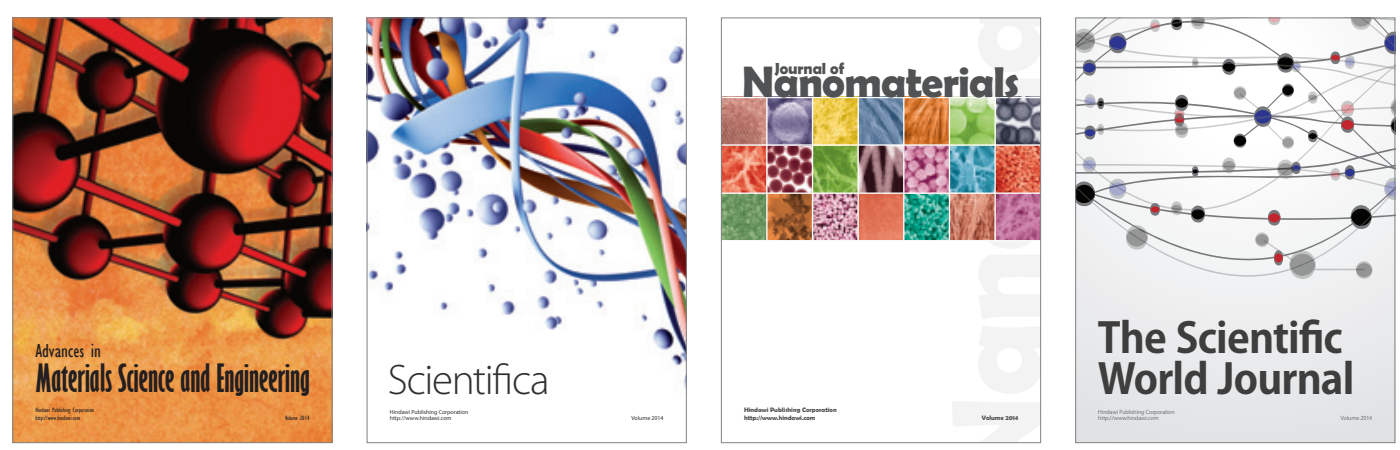

\section{The Scientific World Journal}
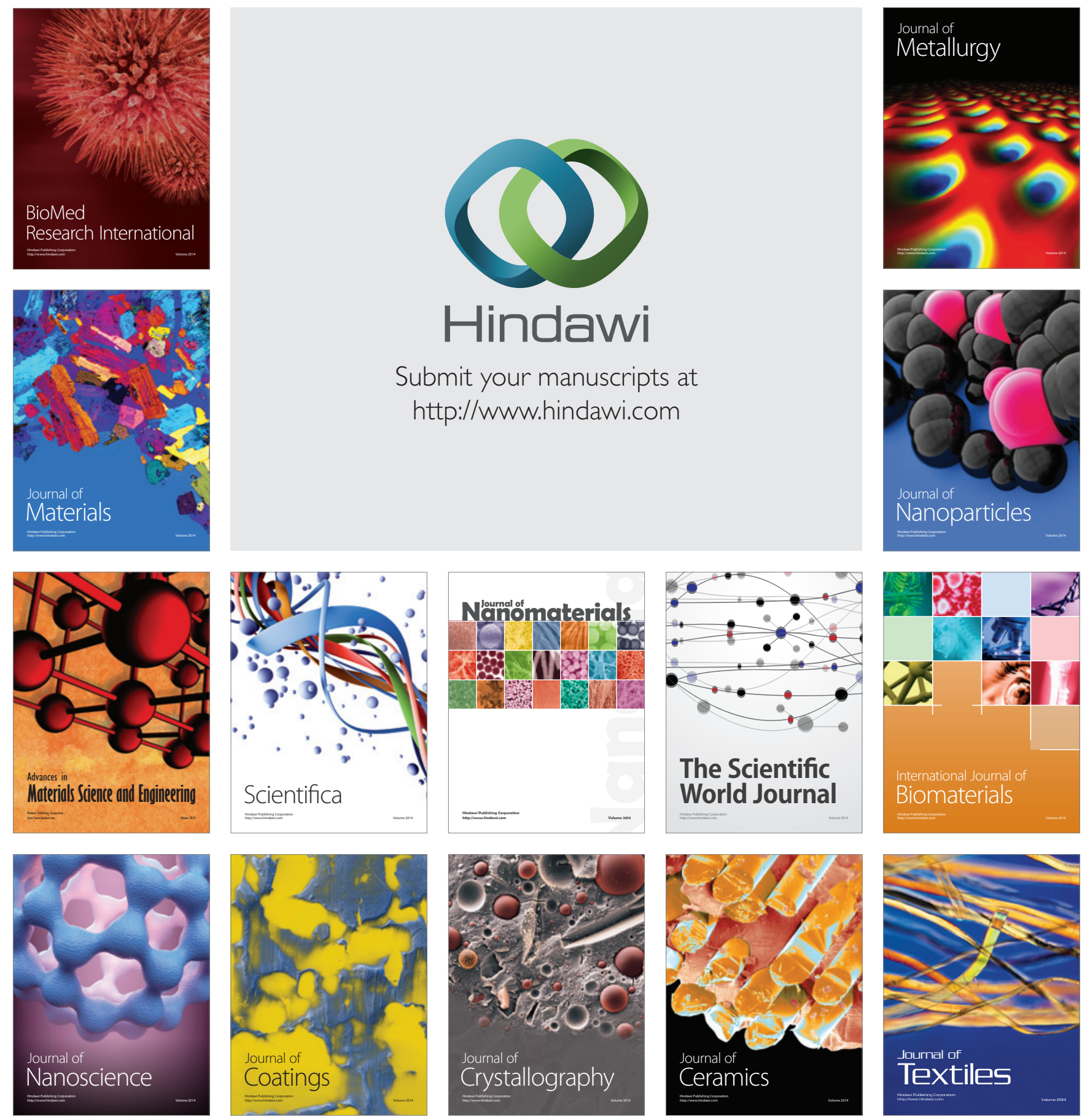